\title{
Impact de quelques substances polluantes (éléments traces métalliques et hydrocarbures aromatiques polycycliques) sur les communautés de vers de terre des bordures de l'autoroute du nord en Côte d'Ivoire.
}

\author{
Mamadou Toure 1, 2, Seydou Tiho 1,2, N'guetta Moïse Ehouman 1, 2, Kouakou Gains Kpan Kpan 1, 2. \\ 1. UFR des Sciences de la Nature (SN), Université Nangui Abrogoua, 02 BP 801 Abidjan 02, Côte d'Ivoire. \\ 2. Unité de Recherche en Ecologie et Biodiversité (UREB). \\ Auteur pour les correspondances : tourexham@yahoo.fr
}

Original submitted in on $25^{\text {th }}$ October 2016. Published online at www.m.elewa.org on $30^{\text {th }}$ November 2016

http://dx.doi.org/10.4314/jab.v107i1.7

\begin{abstract}
RESUME
Objectifs : Evaluer l'impact des substances polluantes, éléments traces métalliques (ETM) et hydrocarbures aromatiques polycycliques (HAP), sur les communautés de vers de terre des bordures de l'autoroute du nord en Côte d'Ivoire.

Méthodologie et résultats : Les substances polluantes ETM et HAP et les vers de terre ontété échantillonnés à partir de monolithes de type TSBF (Tropical Soil Biology and Fertility) de dimension $50 \mathrm{~cm} \times 50 \mathrm{~cm} \times 30 \mathrm{~cm}$, réalisés le long de 10 transects reliant chacun les deux bords de l'autoroute du Nord en Côte d'Ivoire, en passant par le terre-plein central. Le long des bordures de cette autoroute, 8 ETM et les HAP ont été détectés et quantifiés et 19 espèces de vers de terre collectées. II est ressortit que les concentrations des différentes substances polluantes ETM et HAP totaux baissent au fur et à mesure qu'on s'éloigne de la chaussée tandis que la densité totale des vers de terre a tendance à augmenter. Ces deux composantes se sont également montrées inversement proportionnelle en termes de colocation spatiale de part et d'autre de l'autoroute. L'analyse factorielle des correspondances (AFC) a montré que l'ensemble des facteurs écologiques propres aux monolithes réalisés le long des bordures de l'autoroute expliquent à 44,89\% la distribution des vers de terre. L'analyse factorielle des correspondances (ACC) a cependant montré que les substances polluantes ETM et HAP totaux expliquent à eux seules $92,27 \%$ de la variance des vers de terre expliquée par l'AFC. Les 2 principaux axes canoniques de cette ACC qui renseignent le plus sur la relation entre les vers de terre et les substances polluantes (ETM et HAP) ont été défini par le plomb, le thallium, le cuivre et le nickel.

Conclusion et applications des résultats : Le long des bordures de l'autoroute du Nord en Côte d'Ivoire, les ETM et HAP totaux ont dans l'ensemble une distribution inverse à celle de la densité des vers de terre et sont aussi inversement proportionnelles en termes de colocation. Le plomb, le thallium, le cuivre et le nickel sontles principaux polluants qui impactent négativement la distribution des communautés des vers de terre. Ces résultats mettent en évidence une possibilité de choix des vers de terre comme (i) indicateurs de la pollution en ETM et HAP totaux et (ii) organismes biologiques cibles pour les études d'impact environnemental.
\end{abstract}

Mots clés : Vers de terre, Polluants, ETM, HAP, Autoroute du nord, Côte-d'Ivoire 
Impact of a set of pollutants (heavy metals and polycyclic aromatic hydrocarbons) on the earthworm community of the northward motorway's edges in Côte d'Ivoire

\begin{abstract}
Objectives: Assessing the impact of a set of pollutants (heavy metals (HM) and polycyclic aromatic hydrocarbons (PAHs)) on the earthworm community of the northward motorway's edges in Côte d'Ivoire.

Methodology and Results: Heavy metals and polycyclic aromatic hydrocarbons substances and earthworms were sampled using TSBF (Tropical Soil Biology and Fertility) method. Monoliths of $50 \mathrm{~cm} \times 50 \mathrm{~cm}$ and $30 \mathrm{~cm}$ depth were excavated along 10 virtual transects situated on perpendicular axes to the northward motorway and joining both roadside through the central divider strip. Eight pollutants substances (HM and PAHs) were identified and 19 earthworm species collected. On both sides of the northward motorway concentrations of HM and total PAHs substances and the densities of earthworms were found to be inversely distributed. Furthermore, inversely proportional relationships were observed between concentrations of pollutants and densities of earthworms from a roadside to another. The correspondence analysis (CA) showed that all the ecological factors of the monoliths excavated along the highway curbs explain $44.89 \%$ of earthworms' distribution. However, the canonical correspondence analysis (CCA) showed that pollutants ETM and PAHs explain $92.27 \%$ of the earthworms' variance explained by the CA analyses. The 2 main conical axes of this CCA that give main information on the relationship between pollutants (HM and total HAPs) and earthworms have been defined by the lead, thallium, copper and nickel.

Conclusion and application of findings: Along the edges of the highway of the North in Côte d'Ivoire, the distribution of the HM and total PAHs was generally inversed with respect to the density of earthworms and also inversely proportional in terms of collocation. The canonical ordinations showed that the distribution of earthworms along the edges of the highway is almost explained by $\mathrm{HM}$ and the concentrations of PAHs substances. Lead, thallium, copper and nickel are the main pollutants that affect negatively the distribution of earthworm communities. These results indicate an ability to use earthworms as (i) indicators of pollution cause by HM and PAHs and (ii) target biological organisms of environmental impact assessment.
\end{abstract}

Keywords: Earthworms, pollutants, HM, HAPs, Northward motorway's edges, Côte d'Ivoire

\section{INTRODUCTION}

La pollution des sols par les éléments traces métalliques (ETM) et les hydrocarbures aromatiques polycycliques (HAP) représente un problème de société. En effet, ces substances sont naturellement présentes à de faibles concentrations dans l'environnement (Baize, 1997 ; Wcislo, 1998). Par contre, l'augmentation de leurs concentrations est généralement liée à de nombreuses activités humaines parmi lesquelles le trafic routier occupe une place de choix (Trombulak \& Frissell, 2000 ; Jakub \& Marcin, 2016). La pollution des sols par les ETM et les HAP engendrée par le trafic routier représente une fraction importante de la pollution chronique provenant des véhicules en circulation et de l'usure des équipements de la route (Pagotto, 1999 ; Durand, 2003). Ainsi, le long des axes routiers et autoroutiers, ces substances sont d'abord émises vers l'atmosphère et retombent ensuite sous forme de dépôts secs ou humides sur la voie et ses alentours (Durand, 2003). Les ETM et les HAP étant non biodégradables, en s'accumulant dans le sol, altèrent les processus biologiques naturels (Forman \& Alexander, 1998 ; Trombulak \& Frissell, 2000) et peuvent entrer, par l'intermédiaire des végétaux dans la chaîne trophique (Atayese et al., 2009; Ducoulombier \& Rychen, 2009) et constituer une menace pour la santé animale et humaine. Selon des études menées par des auteurs tels que Panisset et al., (2003) ; WHO (2013), l'accumulation des ETM et HAP dans l'organisme des animaux et des humains entraine de graves affections notamment, les maladies neurologiques, le cancer, l'ostéomalacie, l'hydrargyrisme, la néphrite tubulaire et les troubles de la croissance chez l'enfant. Dans de nombreux pays d'Afrique sub-saharienne, la démographie galopante combinée à une 
urbanisation accélérée, ont eu pour conséquence l'augmentation du trafic routier. Ce trafic est par ailleurs marqué par l'utilisation de véhicules d'occasion importés, l'absence de contrôles techniques fiables des véhicules ainsi que l'utilisation croissante des engins à deux roues et de carburants de mauvaise qualité (Banque mondiale, 2003; N'Guessan, 2010; Public Eye, 2016). Ces insuffisances ont pour corollaire l'augmentation drastique des concentrations en substances polluantes notamment les ETM et les HAP au niveau des sols des bordures de route et autoroute. Cependant, cette problématique a été très peu abordée dans les études conduites dans ces pays sub-sahariens, notamment en Côte d'Ivoire (Addo et al., 2012 ; Akan et al., 2013). Il est donc important de se pencher sur l'impact des polluants émis par le trafic routier sur la biodiversité des bordures de route en milieu sub-saharien afin d'anticiper sur les conséquences des ETM et des HAP sur la biocénose qui jalonne les bordures de routes et autoroute de cette région. En effet, de nombreuses études réalisées sur les sols pollués ont révélé que la densité, la capacité de survie et la reproduction des organismes endogés, tels que les vers de terre, sont négativement affectées par les ETM et HAP (Pizl \& Josens, 1995 ; Contreras-Ramos et al.,

\section{MATERIEL ET METHODES}

Site d'étude : Cette étude a été réalisée le long de l'autoroute reliant les districts d'Abidjan et de Yamoussoukro, respectivement capitales, économique et politique de la Côte d'Ivoire. Cette autoroute est subdivisée en deux tronçons dont l'ancien tronçon reliant le district d'Abidjan et la ville de Singrobo qui a été mis en service en 1982 et le nouveau tronçon reliant la ville de Singrobo et le district de Yamoussoukro qui a été mis en service en décembre 2013. Le nouveau tronçon n'étant pas encore officiellement inauguré au moment de l'exécution de cette étude, les travaux ont été effectués le long de l'ancien tronçon (Abidjan-Singrobo), localisé entre les $5^{\circ} 18$ et $6^{\circ} 80$ de latitude Nord et $4^{\circ} 00$ et $5^{\circ} 50$ de longitude Ouest.
2006). Pourtant, ces organismes sont des acteurs très importants dans la vie et la fertilité des sols (Brown et al., 2000). Qualifiés d'ingénieurs de l'écosystème, les vers de terre génèrent des changements directs ou indirects sur les ressources d'autres espèces en impactant les compartiments biotiques et abiotiques (Jones et al., 1994 ; 1997). De ce fait, leur abondance spécifique dans les sols influence d'autres organismes tels que les plantes (Huynh, 2009), les micro-organismes (Blouin et al., 2013) et les invertébrés (Lavelle, 1996). Aussi, la présence des vers de terre dans le sol est-il synonyme d'un bon état de santé de ce sol (Conder et al., 2001). La présente étude a pour objectif d'évaluer l'impact des substances polluantes (ETM et HAP) sur les communautés de vers de terre le long des bordures de l'autoroute du Nord en Côte d'Ivoire. Pour aborder cette étude, nous avons postulé que les substances polluantes ETM et HAP totaux émanant du trafic automobile, influencent négativement la distribution des communautés de vers de terre vivant à proximité des routes. Ce travail a pour objectifs spécifiques d'analyser la distribution des substances polluantes (ETM et HAP totaux) et la diversité des vers de terre le long des bordures de l'autoroute du nord en Côte d'Ivoire afin d'en déduire les relations existant entre ces deux composantes.

Dispositif d'échantillonnage : Le long du tronçon choisi (Abidjan-Singrobo), sur une distance de $90 \mathrm{~km}$, dix transects linéaires reliant les deux bords de l'autoroute, en passant par le terre-plein, ont été établis de sorte à ce qu'ils soient distants de $10 \mathrm{~km}$ les uns des autres afin d'éviter l'influence spatiale de l'un sur l'autre. Sur chacun de ces transects, 11 monolithes de type TSBF (Tropical Soil Biology and Fertility), de $50 \mathrm{~cm} \times 50 \mathrm{~cm}$ sur $30 \mathrm{~cm}$ de profondeur ont été creusés. Cinq monolithes ont été creusés de part et d'autre de la chaussée tandis qu'un seul l'a été au niveau du terre-plein central (TPC). Les 05 monolithes creusés de part et d'autre de la chaussée l'ont été de ce fait sur un transect linéaire virtuel de $200 \mathrm{~m}$ de long à équidistance de $50 \mathrm{~m}$ comme l'indique la Figure 1. 


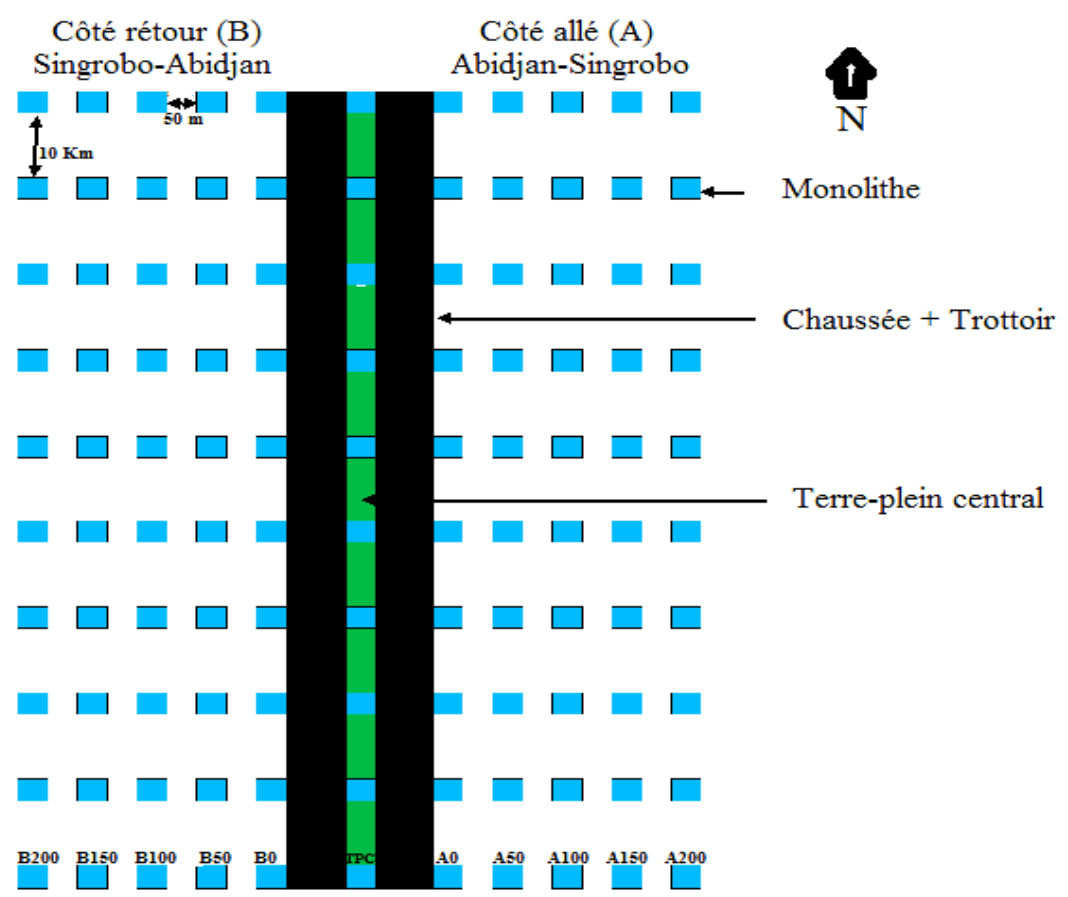

Figure 1 : Dispositif d'échantillonnage

TPC = terre-plein central; $\mathbf{A} 0=$ côté allé juste après le trottoir; $\mathbf{A} 50=$ côté allé 50 mètres du trottoir; $\mathbf{A} 100=$ côté allé 100 mètres du trottoir; $\mathbf{A} 150$ = côté allé 150 mètres du trottoir; $\mathbf{A} \mathbf{2 0 0}=$ côté allé 200 mètres du trottoir; $\mathbf{B} \mathbf{0}=$ côté retour juste après le trottoir; $\mathbf{B} 50=$ côté retour 50 mètres du trottoir; $\mathbf{B} 100=$ côté retour 100 mètres du trottoir; $B 150=$ côté retour 150 mètres du trottoir; $\mathbf{B 2 0 0}=$ côté retour 200 mètres du trottoir.

Fouille des monolithes, collecte et identification des espèces de vers de terre : Chaque monolithe creusé est fouillé manuellement de manière stratifiée $(0-10 \mathrm{~cm} ; 10$ $20 \mathrm{~cm}$ et $20-30 \mathrm{~cm}$ ) à la recherche de vers de terre selon Lavelle (1978). Des échantillons de sol sont également prélevés au niveau de chaque strate de fouille du monolithe et mélangés pour en sortir un échantillon composite. Les vers de terre collectés ont été conservés dans des bocaux dûment étiquetés contenant du formol dilué à $4 \%$. Les bocaux sont ensuite transportés au laboratoire où une identification sur la base de la morphologie (forme, taille, longueur des segments) des individus est faite à l'aide des clés de détermination d'Omodeo et Vaillaud (1967) et de Cszudi et Tondoh (2007).

Détection et quantification des substances polluantes : Les quantités des différents éléments traces métalliques (ETM) et des hydrocarbures aromatiques polycycliques (HAP) ont été mesurées à partir des échantillons composites de sol réalisés à partir des monolithes creusés le long des différents transects. Les ETM ont été détectés et quantifiés par la méthode de la Spectrométrie à Diffusion d'Energies (EDS) à partir d'un
Microscope Electronique à Balayage (MEB FEG Supra 40 VP Zeiss), au Laboratoire du Département d'Analyse et de Recherche de la Société Nationale d'Opération Pétrolière de Côte d'Ivoire (PETROCl). Ainsi, l'échantillon de sol est finement broyé dans un mortier en porcelaine puis tamisé sur un tamis de maille $250 \mu$. Environ $10 \mathrm{mg}$ de la poudre obtenue après tamisage sont prélevés et étalés sur un plot apprêté avec du carbone adhésif à double face. L'appareil effectue une mesure de l'énergie de transition des électrons au niveau des nuages électroniques des séries $K, L$ et $M$ des atomes de l'échantillon ce qui permet d'identifier sa composition chimique. Les substances observées ont ensuite été quantifiées à l'aide d'un spectromètre d'absorption atomique SPECTRA 110 (VARIAN). Cette opération est réalisée après digestion de $0,5 \mathrm{~g}$ de chaque échantillon dans $5 \mathrm{ml}$ d'acide nitrique $\left(\mathrm{HNO}_{3}\right)$ à $65 \%, 2 \mathrm{ml}$ d'acide sulfurique $\left(\mathrm{H}_{2} \mathrm{SO}_{4}\right)$ à $98 \%$ et $1 \mathrm{ml}$ de peroxyde d'hydrogène $\left(\mathrm{H}_{2} \mathrm{O}_{2}\right)$ à $30 \%$ selon Kpan Kpan et al., (2014). Les HAP ont été extraits avec un mélange acétonitrile / acétone, puis purifiés sur des cartouches de phase greffée en $\mathrm{C} 18$ ou sur des cartouches de Fluorisil. La détermination de la teneur en HAP individuel après 
séparation a été réalisée par le biais du HPLC en mesurant la fluorescence à des longueurs d'onde d'excitation et d'émission différentes ou mesurant l'absorption de l'UV par les HAP. Ces différentes mesures ont été effectuées au Laboratoire National d'Appui au Développement Agricole de Côte d'Ivoire (LANADA). Par la suite, l'étude à été effectuée avec la somme des HAP (HAP totaux) identifiés (distribution et co-distribution spatiale avec les vers de terre).

Analyses statistiques : La distribution de la teneur des substances polluantes détectées et de la densité des vers de terre au fur à mesure qu'on s'éloigne de la chaussée a été représentée grâce à la fonction buble du package gstat du programme $\mathrm{R}$. Cette fonction a permis de faire des cartes sur lesquelles, les concentrations des substances polluantes et les densités des vers de terre obtenues au niveau des différents points d'échantillonnage le long des transects sont matérialisées par des cercles ou patch. Les comparaisons de moyennes des teneurs des substances polluantes détectées et des densités des vers de terre entre les points d'échantillonnage le long des transects ont été effectuées à l'aide du test non paramétrique de KruskalWallis grâce au logiciel STATISTICA 7.1. La codistribution spatiale de la teneur des différentes substances polluantes détectées et la densité des vers de terre collectés a été présentée grâce à la fonction xplot du package lattice graphics du programme de R. Cette

\section{RESULTATS}

Diversité et distribution des vers de terre : Au total, 19 espèces de vers de terre ont été collectées. II s'agit des espèces Agastrodrillus multivesiculatus, Chuniodrilus palustris, Chuniodrilus vouattuoxi, Chuniodrilus zielae, Dichogaster agilis, Dichogaster arboricola, Dichogaster baeri, Dichogaster bolani, Dichogaster saliens, Dichogaster sp1, Dichogaster sp2, Dichogaster terraenirae, Eudrilus eugeniae, Hyperiodrilus africanus, Millsonia ghaneansis, Millsonia lamtoiana, Millsonia omodeoi, Millsonia schelegilli et Stuhlmannia prolifera. La distribution de la densité par monolithe de ces vers de terre le long des différents transects a montré que de part et d'autre de l'autoroute, les plus fortes densités (larges méthode a permis de présenter au niveau d'un même point d'échantillonnage les concentrations des substances polluantes et la densité des vers à partir de différents icônes superposées. Ainsi, la grandeur de chaque composante est proportionnelle à la taille de l'icône qui la représente. Les analyses statistiques multivariées exécutées à l'aide du package ade4 du logiciel $R$, ont consisté en l'analyse de l'interaction entre les différentes substances polluantes et la communauté des vers de terre collectés à partir de différentes méthodes d'ordination développées par Ter Braak (1986, 1987) et Lebreton et al., (1988), notamment les analyses factorielles des correspondances (AFC) et canoniques des correspondances (ACC). L'AFC a d'abord été utilisée pour évaluer la variabilité des vers de terre expliquée par l'ensemble des facteurs écologiques propre au milieu d'échantillonnage (bords de l'autoroute), suivi d'une application de l'ACC pour la mesure des relations entre les vers de terre et les variables environnementales (substances polluantes détectées). Cette analyse impose une contrainte linéaire (Ter Braak, 1986) entre les variables (substances polluantes détectées) et les axes principaux. Un test de permutation de Monte-Carlo a été appliqué pour déterminer la signification statistique des axes de l'ACC. Puis, les coefficients canoniques de corrélation ont été utilisés pour déterminer les substances polluantes contribuant significativement à la construction des principaux axes canoniques.

cercles) ont été observées aux points d'échantillonnage les plus éloignées de la chaussée (Figure 2). Par contre, de faibles densités (petits cercles et points) des vers de terre, à proximité immédiate de la chaussée ont été observées de manière générale. En somme, au fur et à mesure que l'on s'éloigne de la chaussée une tendance à l'augmentation des densités de vers de terre a été notée. Les densités par monolithe des vers à proximité immédiate de la chaussée se sont montrées significativement différentes de celles qui sont les plus éloignées (test de Kruskal-Wallis, $p<0,05$ ) (Tableaux $1 a$ et $1 b)$. 


\section{Distribution de la densité des vers de terre}

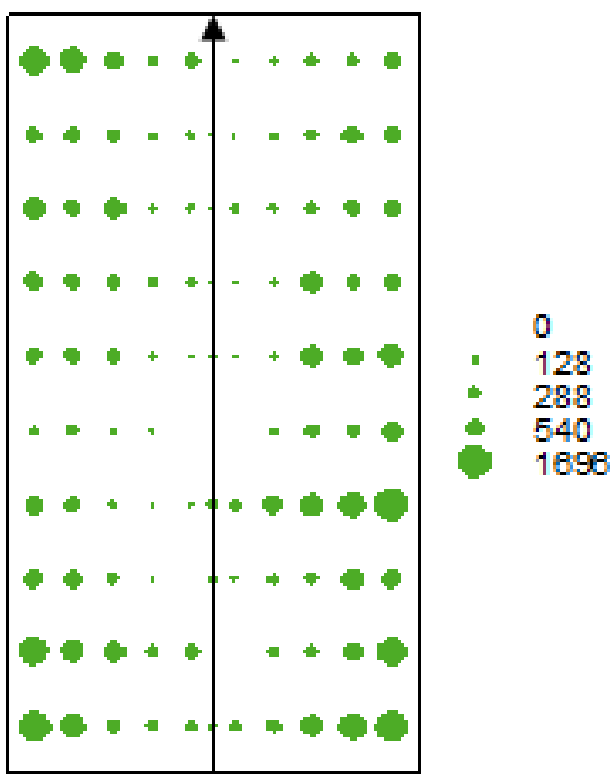

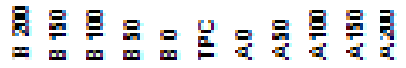

Figure 2 : Carte de la distribution des densités de vers de terre par monolithe (nombre d'individus $/ \mathrm{m}^{2}$ ) le long des 10 transects d'échantillonnage

TPC = terre-plein central; $\mathbf{A} 0$ = côté allé juste après le trottoir; $\mathbf{A} 50$ = côté allé 50 mètres du trottoir; $\mathbf{A} 100=$ côté allé 100 mètres du trottoir; $\mathbf{A} 150$ = côté allé 150 mètres du trottoir; $\mathbf{A} 200$ = côté allé 200 mètres du trottoir; $\mathbf{B} \mathbf{0}=$ côté retour juste après le trottoir; $\mathbf{B} 50=$ côté retour 50 mètres du trottoir; $\mathbf{B} 100=$ côté retour 100 mètres du trottoir; $\mathbf{B} 150=$ côté retour 150 mètres du trottoir; $\mathbf{B 2 0 0}=$ côté retour 200 mètres du trottoir.

Tableau 1a : Résultats du test de Kruskal-Wallis (valeurs $p$ ) pour la distribution des densités de vers de terre du côté allé (A) de l'autoroute (Abidjan-Singrobo)

\begin{tabular}{|c|c|c|c|c|c|c|}
\hline \multicolumn{7}{|c|}{ Côté allé (A) } \\
\hline & TPC & $\mathrm{AOm}$ & A $50 \mathrm{~m}$ & A $100 \mathrm{~m}$ & A $150 \mathrm{~m}$ & A $200 \mathrm{~m}$ \\
\hline TPC & - & & & & & \\
\hline $\mathrm{AOm}$ & 1,000000 & - & & & & \\
\hline A $50 \mathrm{~m}$ & 1,000000 & 1,000000 & - & & & \\
\hline A $100 \mathrm{~m}$ & $0,028631^{* *}$ & $0,009578^{\star *}$ & 1,000000 & _ & & \\
\hline A $150 \mathrm{~m}$ & $0,001857^{* *}$ & $0,000518^{* *}$ & 0,329862 & 1,000000 & - & \\
\hline A $200 \mathrm{~m}$ & $0,000030^{* *}$ & $0,000007^{* *}$ & $0,016605^{\star *}$ & 1,000000 & 1,000000 & _- \\
\hline
\end{tabular}

** : différence significative $(\mathrm{p}<0,05) ; \mathrm{TPC}=$ terre-plein central; $\mathbf{A} \mathbf{0}=$ côté allé juste après le trottoir; $\mathbf{A} 50=$ côté allé 50 mètres du trottoir; $\mathbf{A} 100$ = côté allé 100 mètres du trottoir; $\mathbf{A} 150$ = côté allé 150 mètres du trottoir; $\mathbf{A 2 0 0}=$ côté allé 200 mètres du trottoir. 

vers de terre des bordures de l'autoroute du nord en Côte d'Ivoire.

Tableau 1a : Résultats du test de Kruskal-Wallis (valeurs $p$ ) pour la distribution des densités de vers de terre du côté retour $(B)$ de l'autoroute (Singrobo-Abidjan)

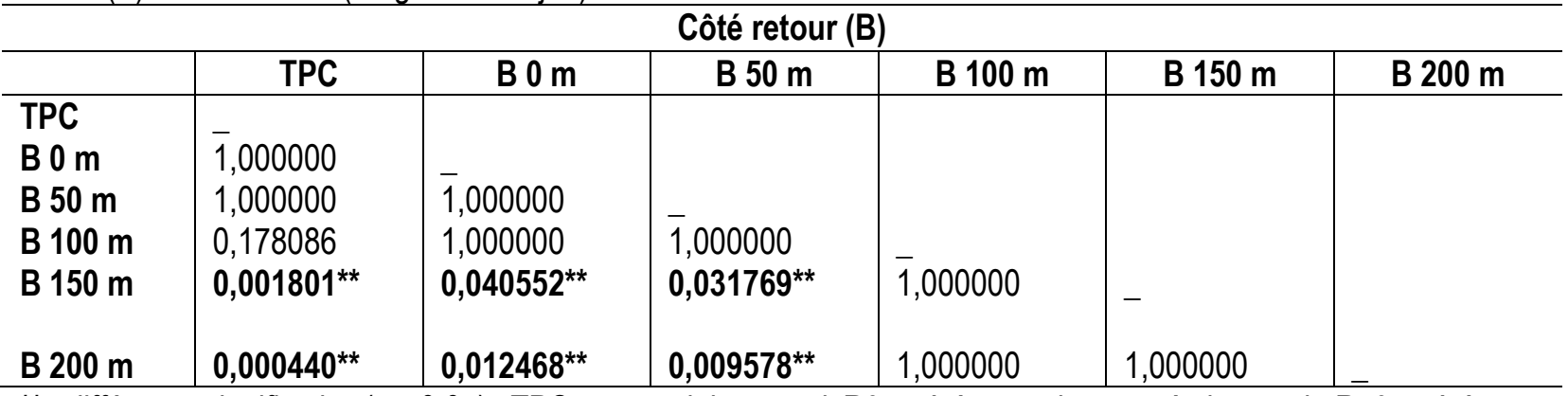

${ }^{* *}$ : différence significative $(p<0,05) ;$ TPC = terre-plein central; $\mathbf{B} \mathbf{O}=$ côté retour juste après le trottoir; $\mathbf{B} 5 \mathbf{5 0}=$ côté retour 50 mètres du trottoir; $\mathbf{B} 100=$ côté retour 100 mètres du trottoir; $\mathbf{B} 150=$ côté retour 150 mètres du trottoir; $\mathbf{B} 200=$ côté retour 200 mètres du trottoir.

Détection et quantification des substances polluantes le long des transects d'échantillonnage : Huit ETM ont été détectées le long des 10 transects d'échantillonnage, à savoir le cobalt (Co), le chrome $(\mathrm{Cr})$, le cuivre $(\mathrm{Cu})$, le manganèse $(\mathrm{Mn})$, le nickel $(\mathrm{Ni})$, le plomb $(\mathrm{Pb})$, le thallium (Th) et le zinc (Zn). Seize substances d'HAP de la liste de I'US-EPA ont été détectées: le naphtalène, l'acénaphtalène, l'acénaphtylène, le fluorène, le phénanthrène, l'anthracène, le fluoranthène, le pyrène, le benzo (a) anthracène, le chrysène, le benzo (b) fluoranthène, le benzo $(k)$ fluoranthène, le benzo $(a)$ pyrène, le benzo $(g, h$, i) pyrène, le dibenzo (ah) anthracène et l'indéno $(1,2,3, \mathrm{~cd})$ pyrène. La répartition des ETM et de l'ensemble des 16 HAP (HAP totaux) a été observée le long des transects (Figure 3). En effet, de fortes concentrations (grands cercles sur la figure 3 ) des substances polluants (ETM et HAP totaux) ont été détectées à proximité immédiate de la chaussée. Par contre, de faibles concentrations de ces substances polluantes (petits cercles et points) ont été notées au fur et à mesure que les points d'échantillonnage s'éloignaient de la chaussée. De manière générale, pour toutes les substances détectées et quantifiées, une tendance à la baisse a été observée avec l'éloignement progressif de la chaussée (test de Kruskal-Wallis, $p<0,05$ ).
Répartition du chrome

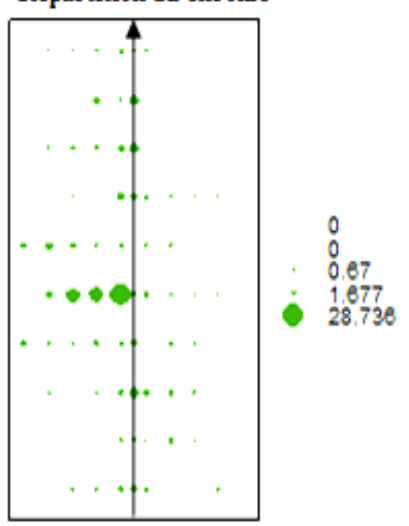

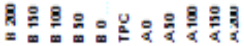

Répartition du cobalte

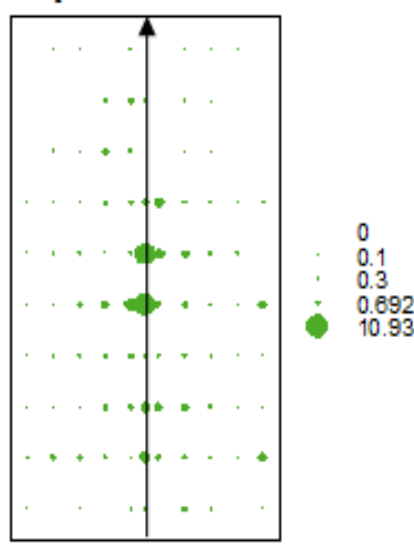

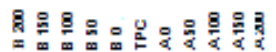

Répartition du cuivre

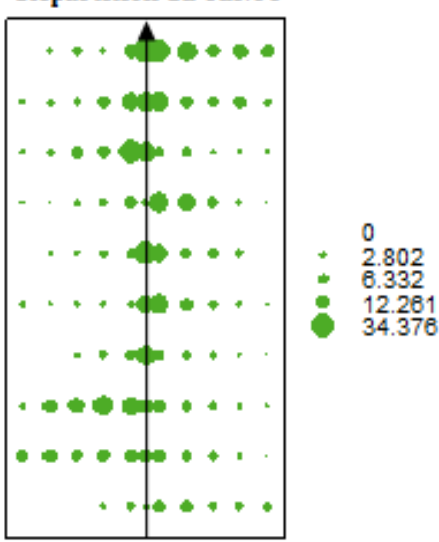

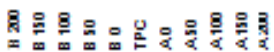


Répartition du plomb

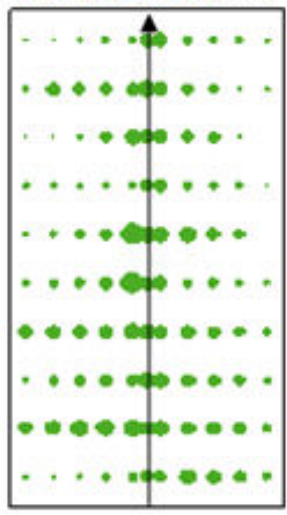

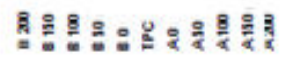

Répartition du nickel

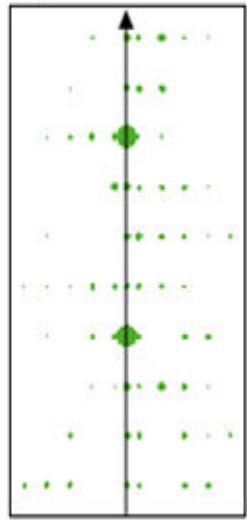

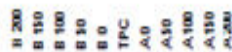

Répartition du manganèse

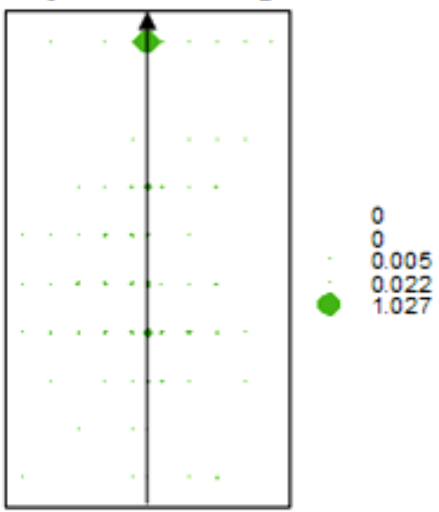

을 을
Répartition du thallium

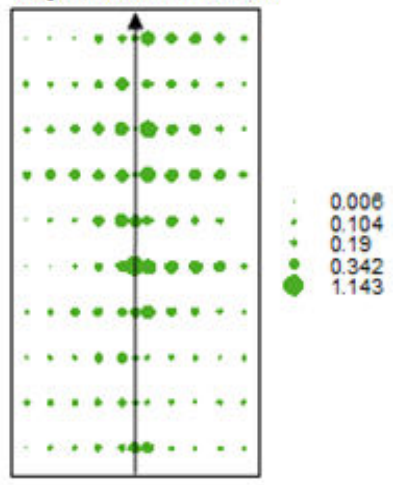

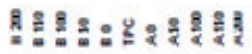

0.016

14.523

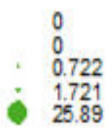

Répartition du zinc

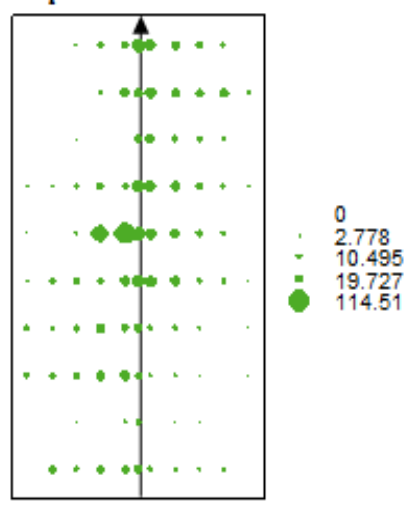

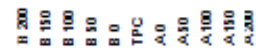

Répartition des HAP totaux

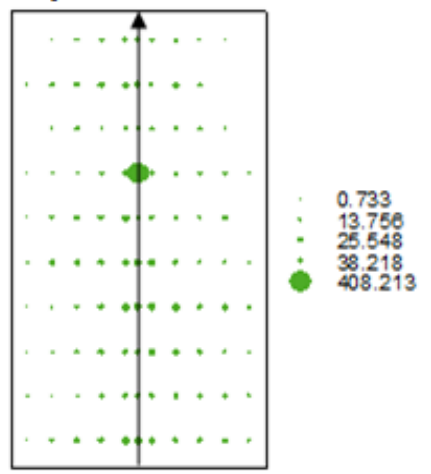

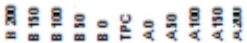

Figure 3 : Cartes de la répartition des substances polluantes $(\mathrm{mg} / \mathrm{kg})$ le long des 10 transects d'échantillonnage TPC = terre-plein central; $\mathbf{A} 0=$ côté allé juste après le trottoir; $\mathbf{A} 50=$ côté allé 50 mètres du trottoir; $\mathbf{A} 100=$ côté allé 100 mètres du trottoir; $\mathbf{A} 150=$ côté allé 150 mètres du trottoir; $\mathbf{A} 200=$ côté allé 200 mètres du trottoir; $\mathbf{B} \mathbf{0}=$ côté retour juste après le trottoir; $\mathbf{B} 50=$ côté retour 50 mètres du trottoir; $\mathbf{B} 100=$ côté retour 100 mètres du trottoir; B150 = côté retour 150 mètres du trottoir; $\mathbf{B 2 0 0}=$ côté retour 200 mètres du trottoir.

Impact des concentrations des substances polluantes (ETM et HAP totaux) sur les densités des vers de terre le long des transects d'échantillonnage : Les concentrations des différentes substances polluantes (ETM et HAP totaux) se sont révélées inversement proportionnelles à la densité des vers de terre le long des transects d'échantillonnage (Figure 4). En effet, plus les concentrations respectives des substances polluantes sont fortes, moins denses sont les vers de terre sur chaque transect. 

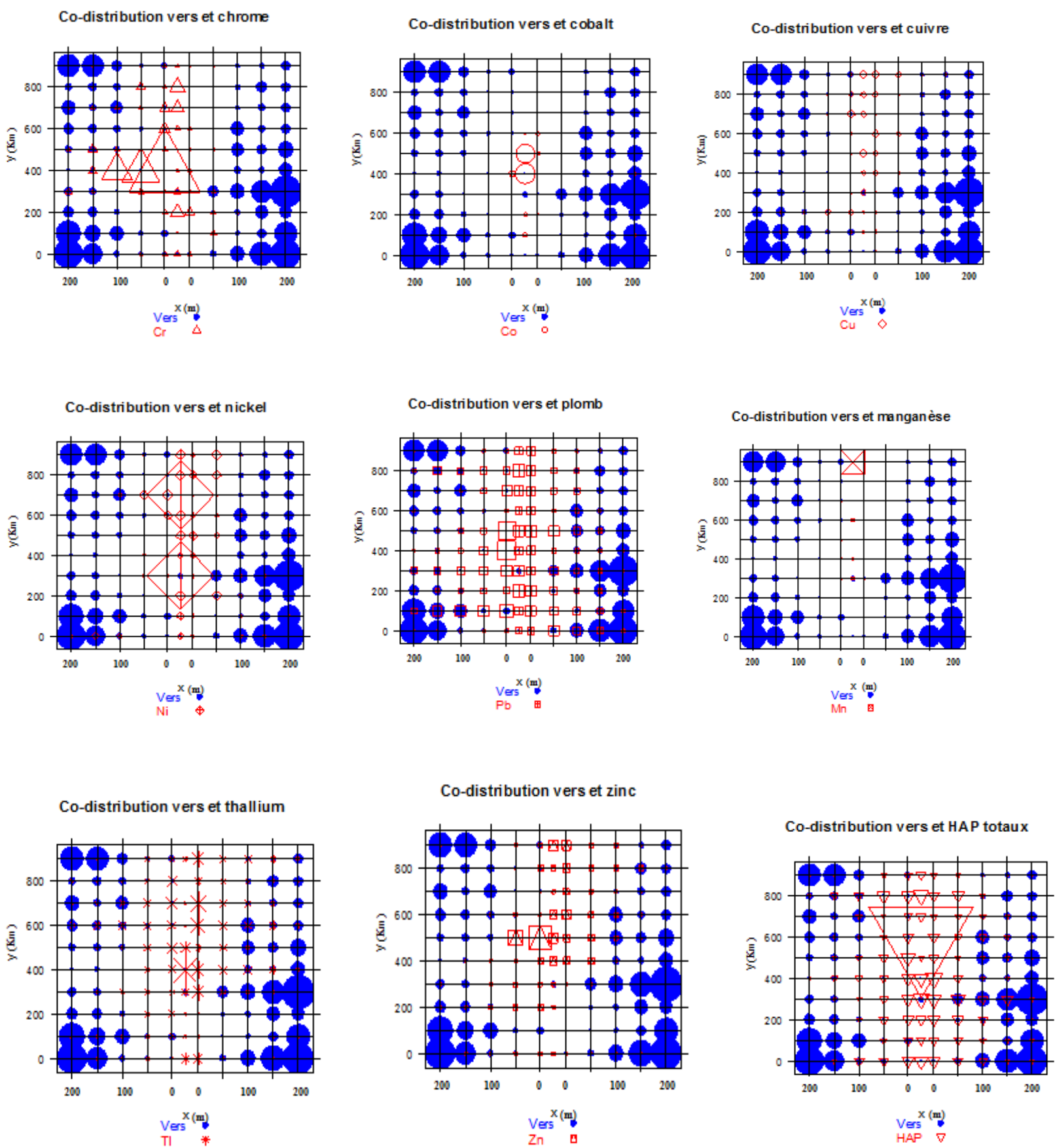

Figure 4 : Impact des concentrations respectives des substances polluantes (ETM et HAP totaux) sur les densités des vers de terre le long des transects d'échantillonnage.

\section{Analyses multivariées}

Analyse factorielle des correspondances: Les résultats de l'analyse factorielle des correspondances (AFC) réalisée pour les vers de terre a montré que les 4 premiers axes qui portent l'essentiel des informations sur l'ensemble des facteurs écologiques propres aux monolithes réalisés le long des bordures de l'autoroute et susceptibles d'influencer la distribution des vers ont expliqué à $44,89 \%$, la variance totale de la matrice des vers de terre collectés (Tableau 2). 
Tableau 2 : Analyse factorielle de correspondance avec les vers de terre

Valeurs propres

Pourcentage cumulé de la variance des vers de terre

Inertie totale

\begin{tabular}{|l|l|l|l}
$\mathbf{A x 1}$ & $\mathbf{A x 2}$ & $\mathbf{A x 3}$ & $\mathbf{A x 4}$ \\
\hline 0,401 & 0,272 & 0,181 & 0,174 \\
17,49 & 29,36 & 37,28 & 44,89
\end{tabular}

2,291

Analyse canonique des correspondances: Les résultats de l'ACC ont montré que les 4 premiers axes canoniques qui renseignent le plus sur la relation entre les vers de terre et les substances polluantes ETM et HAP totaux ont expliqués $92,27 \%$ de la variance de la matrice des vers de terre extraite par l'AFC (Tableau 3a). Cependant, les 2 premiers axes canoniques qui portent l'essentielle des informations sur la relation ont été définis par le gradient du nickel et du plomb pour l'axe 1 et par celui du cuivre, du nickel, du plomb et du thallium pour l'axe 2 (Tableau 3b). Le test de permutation de Monte Carlo a montré que le long des bords de l'autoroute, les substances polluantes ont produit une ordination dans laquelle les premiers axes et tous les autres axes canoniques sont significatives $(p<0,05)$.

Tableau 3 a : Analyse canonique des correspondances

\begin{tabular}{l|c|c|c|c}
\hline & Ax1 & Ax2 & Ax3 & Ax4 \\
\hline Valeur propres & 0,252 & 0,141 & 0,045 & 0,027 \\
$\begin{array}{l}\text { Pourcentage de la variance des vers de } \\
\text { terre et des substances polluantes }\end{array}$ & 50,107 & 27,85 & 8,883 & 5,404 \\
$\begin{array}{l}\text { Pourcentage cumulé de la variance des } \\
\text { vers de terre et des substances polluantes }\end{array}$ & 50,11 & 77,98 & 86,86 & $\mathbf{9 2 , 2 7}$ \\
Inertie totale & & & \\
\hline
\end{tabular}

Tableau $3 \mathbf{b}$ : Coefficients canoniques des différentes substances polluantes (ETM et HAP totaux) sur les deux premiers axes

\begin{tabular}{c|c|c|c|c|c|c|c|c|c}
\hline & Chrome & Cobalt & Cuivre & HAP & Manganèse & Nickel & Plomb & Thallium & Zinc \\
\cline { 2 - 9 } Axe1 & 0,268 & 0,092 & 0,226 & 0,055 & 0,176 & $\mathbf{0 , 6 2 6}$ & $\mathbf{0 , 6 1 3}$ & 0,482 & 0,289 \\
Axe 2 & 0,430 & 0,312 & $\mathbf{0 , 7 5 1}$ & 0,379 & 0,471 & $\mathbf{0 , 6 9 5}$ & $\mathbf{0 , 5 2 7}$ & $\mathbf{0 , 5 4 5}$ & $\mathbf{0 , 4 7 8}$ \\
\hline
\end{tabular}

\section{DISCUSSION}

Les ETM et HAP totaux détectés et quantifiés de part et d'autre de l'autoroute du Nord de la Côte d'Ivoire, ont montré de fortes concentrations à proximité immédiate de la chaussée et de faibles concentrations au fur et à mesure que l'on s'éloigne de celle-ci. Ce résultat indique que le trafic automobile sur la chaussée serait en grande partie à l'origine de cette quantité importante de ces substances polluantes de part et d'autre de celle-ci. Et qu'au cours de leur transport respectif, le vent (Contat et al., 1991) et/ou le ruissellement des eaux de pluies
(Pagotto, 1999), perpendiculairement à la chaussée joueraient un rôle dans la diminution des concentrations de ces substances polluantes au fur à mesure que l'on s'éloigne de la chaussée. En effet, ces substances (ETM et HAP) proviennent principalement de l'usure de la chaussée, l'abrasion et corrosion des métaux constitutifs des véhicules (freins, carrosserie), l'usure des pneumatiques et des pots catalytiques, l'émission due aux gaz d'échappement et la corrosion des barrières de sécurité (Pagotto, 1999 ; Promeyrat, 2001). Selon 
Deletraz \& Paul (1998), les ETM ont une zone de forte influence variant entre 30 à 150 mètres de la chaussée et les HAP ont un important dépôt qui s'effectue dans la zone des 100 premiers mètres bordant la chaussée. Par ailleurs, selon Tong et al., (2016), la présence d'une importante quantité de végétaux le long des bordures des routes, comme c'est le cas de notre site d'étude (zone pré forestière), engendre également une diminution des concentrations de ces substances polluantes pendant leurs transports à des distances éloignées de la chaussée. Quant aux vers de terre collectés, contrairement aux substances polluantes, une augmentation de leurs densités par monolithe a été observée au fur et à mesure que l'on s'éloigne de la chaussée. Ces densités se sont révélées inversement proportionnelles aux concentrations des différentes substances polluantes en termes de colocation spatiale. Cette observation pourrait s'expliquer par le fait que la survie des vers de terre soit négativement impactée par les fortes concentrations de substances polluantes mesurées à proximité de la chaussée. Ce résultat est en adéquation avec ceux de plusieurs auteurs (Pižl \& Josens, 1995 ; Spurgeon et al., 2000 ; Nahmani et al., 2003 ; Zheng et al., 2008) qui ont montré que les ETM et les HAP réduisent la densité des vers de terre, leur croissance pondérale et leur fertilité. Par ailleurs, l'utilisation du mécanisme d'évitement des poches de pollution du sol pourrait également expliquer cette réduction des densités de vers de terre par monolithe le long des transects d'échantillonnage. Et ce, à travers leur grande capacité de différenciation de la matière organique en fonction de son niveau de contamination comme le suggère Depta et al., (1999). L'AFC a montré que l'ensemble des facteurs écologiques propres aux monolithes réalisés le long des bordures de l'autoroute et qui sont susceptibles d'influencer la distribution des vers de terre ont expliqué à environ $45 \%$ la variance des vers de terre. Par conséquent, la distribution de ces organismes serait en grande partie liée à la présence des substances polluantes et aux conditions stochastiques. En effet, dans l'interprétation du pourcentage de variance mesurée, nous devons tenir compte du fait que ce

\section{CONCLUSION}

L'analyse de la relation existant entre les substances polluantes (ETM et HAP totaux) et les communautés de vers de terre a montré que de part et d'autre de l'autoroute du nord en Côte d'Ivoire, la distribution des substances ETM (cobalt, chrome, cuivre, manganèse, nickel, plomb, thallium et zinc) et des HAP totaux est caractérisée par une baisse de leurs concentrations au pourcentage n'atteint jamais les $100 \%$, car une partie de cette variance est due aux "bruits" des données interprétées comme étant la résultante d'une fluctuation aléatoire des espèces (Gauch, 1982). La structure des données étant résumée, selon cet auteur, dans les premiers axes principaux tandis que les autres résument le bruit des données. Une ordination qui explique seulement un faible pourcentage peut néanmoins contenir une bonne partie de l'information écologique. Cette information a été confirmée par l'ACC qui a montré que la distribution des vers de terre le long des bordures de l'autoroute, est principalement expliquée par les concentrations des substances polluantes ETM et HAP totaux détectées et quantifiées $(92,27 \%)$. Ce résultat est aussi une confirmation de la colocation spatiale observée entre les densités de vers de terre et les concentrations de substances polluantes qui a montré que ces deux composantes sont inversement proportionnelles en termes de colocation. Les résultats de cette ACC ont également montré de fortes corrélations entre les concentrations de nickel, de plomb, de cuivre et de thallium et les densités de vers de terres; ce qui indique qu'ils seraient les principaux polluants entrainant une réduction des densités de vers au niveau des bordures de cette autoroute. En effet, bien qu'étant indispensables à l'état de trace, pour de nombreux processus cellulaires chez les vers de terre, le cuivre et le nickel peuvent se révéler toxiques à forte concentration et avoir des effets néfastes tels que la réduction de leur biomasse et la réduction de la production de cocon (Scott-Fordsmand et al., 1998 ; 2000 ; Maboeta et al., 2004). Il en est de même pour le plomb (Spurgeon \& Hopkin, 1995 ; Davies et al., 2003) et le thallium (Environment Canada, 1996), qui ne jouent toutefois aucun rôle dans les processus cellulaires des vers de terre et s'avèrent plutôt toxiques même à de faibles concentrations pour les organismes biologiques. Ces résultats ont montré le niveau de nuisance que peut avoir les substances polluantes, notamment les ETM, sur les densités des communautés de vers de terre des bordures de l'autoroute du nord en Côte d'Ivoire, par un impact négatif considérable sur la distribution et même probablement la survie de ces organismes.

fur et à mesure qu'on s'éloigne de la chaussée tandis que la densité totale des vers de terre a tendance à augmenter. La colocation spatiale de la densité totale des vers de terre et des concentrations de ces différentes substances polluantes a mis en évidence une relation inversement proportionnelle. Les ordinations canoniques ont montré que la distribution des vers de terre le long 
des bordures de cette autoroute est principalement expliquée par les concentrations des substances ETM et HAP totaux. Le plomb, le thallium, le cuivre et le nickel

\section{REMERCIEMENTS}

Nos remerciements vont à l'endroit du Laboratoire du Département d'Analyse et de Recherche de la Société Nationale d'Opération Pétrolière (PETROCI) et du Laboratoire National d'Appui au Développement Agricole (LANADA). Merci également à Professeurs Kolo YEO et Souleymane KONATE, respectivement Responsable de la Station de Recherche en Ecologie de Lamto et

\section{REFERENCES BIBLIOGRAPHIQUES}

Addo MA, Darko EO, Gordon C, Nyarko BJB, Gbadago JK, 2012. Heavy Metal Concentrations in Road Deposited Dust at Ketu-South District, Ghana. International Journal of Science and Technology 2 (1): 28-39.

Akan JC, Stephen IA, Mohammed Z, Ogugbuaja VO, 2013. Assessment of Heavy Metals, pH, Organic Matter and Organic Carbon in Roadside Soils in Makurdi Metropolis, Benue State, Nigeria. Journal of Environmental Protection 4: 618-628.

Atayese MO, Elgbadon Al, Oluwa KA, Adesodun JK, 2009. Heavy metal contamination of Amaranthus crown along major highways in Lagos, Nigeria. African Crop Science Journal 16 : 225 - 235.

Baize, D, 1997. Teneurs totales en éléments traces métalliques dans les sols (France). INRA Éditions, Paris, $410 \mathrm{p}$.

Banque mondiale, 2003. Initiative sur la qualité de l'air dans les villes d'Afrique sub-saharienne. Rapport d'avancement 1998 - 2002. Document de travail numéro 11. [En ligne] réf. du 13 - 03 2007.

Blouin M, Hodson ME, Delgado EA, Baker G, Brussaard L, Butt KR, Dai J, Dendooven L, Peres G, Tondoh JE, Cluzeau D, Brun JJ, 2013. A review of earthworm impact on soil function and ecosystem services. European Journal of Soil Science 64 : 161-182.

Brown G, Barois I, Lavelle P, 2000. Regulation of soil organic matter dynamics and microbial activity in the drilosphere and the role of interactions with other edaphic functional domains. European Journal of Soil Biology 36: 177-198.

Conder JM, Lanno RP, Basta NT, 2001. Assessment of metal availability in smelter soil using earthworms and chemical extractions. Journal Environnemental Quality 30 : 1231-1237. sont les principaux polluants qui impactent négativement la distribution des communautés des vers de terre.

Directeur de l'Unité de Recherche en Ecologie et Biodiversité de l'Université Nangui Abrogoua, pour le soutien logistique dans la réalisation de cette étude. Nous remercions également les reviewers pour les critiques, corrections et suggestions qui ont contribué à considérablement améliorer la qualité de ce manuscrit.

Contat F, Shariat MH, Stadelmann FX, 1991. "Déposition et accumulation de Plomb le long de quatre secteurs autoroutiers de 1978 à 1988. Évolution en fonction des années, des saisons et de la météorologie. Recherche Agronomique en Suisse 30 (1/2) : 29-43.

Contreras-Ramos SM, Alvarez DB, Dendooven L, 2006. Eisenia fetida increased removal of polycyclic aromatic hydrocarbons from soil. Environmental Pollution 141: 392-401.

Csuzdi C. and Tondoh JE, 2007. New and little-known earthworm species from the Ivory Coast (Oligochaeta: Acanthodrilidae: Benhamiinae and Eudrilidae). Journal of Natural History 41: 25512567.

Davies NA, Hodson ME, Black S, 2003. The influence of time on lead toxicity and bioaccumulation determined by the OECD earthworm toxicity test. Environmental Pollution 121 : 49-54.

Deletraz, G. and Paul, E, 1998. Etat de l'art de l'étude des impacts des transports routiers à proximité des routes et les autoroutes. Le rapport intermédiaire non confidentiel de Mars 1998, ADEME, $144 \mathrm{p}$.

Depta, B, Koscielniak, A, Rozen, A, 1999. Food selection as a mechanism of heavy metal resistance in earthworms. Pedobiologia 42 : 608-614.

Ducoulombier C. and Rychen G, 2009. Les pâtures à proximité des voies routières sont-elles contaminées par les émissions des véhicules? Premiers éléments de réponse. Fourrages 199 : 281-290.

Durand C, 2003. Caractérisation physico-chimique des produits de l'assainissement pluvial. Origine et devenir des métaux traces et des polluants organiques Ph.D. Thesis, Poitiers University, France. 
Environment Canada, 1996. Seedling emergence and earthworm toxicity tests on barium and thallium. Ecosystem Conservation Directorate, Evaluation and Interpretation Branch, Guidelines Division, Ottawa. Unpub.

Forman RTT. and Alexander LE, 1998. Roads and their major ecological effects. Annual Review of Ecology and Systematics 29: 207-231.

Gauch, HGJR, 1982. Multivariate Analysis in Community Structure. Cambridge University Press, Cambridge.

Huynh TMD, 2009. Impact des métaux lourds sur les interactions plante/ ver de terre/ microflore tellurique Océan, Atmosphère. Université ParisEst.

Jakub C. and Marcin S, 2016. Environmental factors affecting soil metals near outlet roads in Poznań, Poland: impact of grain size, soil depth, and wind dispersal. Environmental Monitoring Assessment 188: 323-334.

Jones CG, Lawton JH, Shachak M, 1994. Organisms as ecosystem engineers. Oikos 69: 373-386.

Jones CG, Lawton JH, Shachak M, 1997. Positive and negative effects of organisms as physical ecosystem engineers. Ecology 78: 1946-1957.

Kpan Kpan KG, Yao BL, Dembélé A, Traoré KS, Messoum F, 2014. Contamination des basidiomycètes (Volvariella volvacea et Termitomyces spp) des marchés abidjanais par le plomb, le cadmium, le mercure et le zinc. International Journal of Biological and Chemical Sciences 8: 2356-2366.

Lavelle $P, 1996$. Diversity of soil fauna and ecosystem function. Biology International 33 : 3-16.

Lavelle $P, 1978$. Les vers de terre de la savane de Lamto (Côte d'Ivoire): peuplements, population et fonctions dans les écosystèmes. Thèse d'Etat. Université Paris VI. Publication du Laboratoire de Zoologie de l'ENS n 12.

Lebreton JD, Chessel D, Richardot - Coolet M, Yoccoz N, 1988. L'analyse des relations espèces - milieu par l'analyse canonique des correspondances. I. variable de milieux quantitatifs. Acta oecologia Oecology 9 (2) : 137-151.

Maboeta MS, Reinecke SA, Reinecke AJ, 2004. The relationship between lysosomal biomarker and organismal responses in an acute toxicity test with Eisenia fetida (Oligochaeta) exposed to the fungicide copper oxychloride. Environmental Research 96: 95-101.
Nahmani J, Lavelle P, Lapied E, van Oort F, 2003. Effects of heavy metal soil pollution on earthworm communities in the north of France. Pedobiologia 47 : 663-669.

N'Guessan HJK, 2010. "L'invasion des véhicules d'occasion en transit par le port d'Abidjan : le dynamisme ambivalent d'une activité en plein essor », Les Cahiers d'Outre-Mer, 251 : 365390.

Omodeo P. and Vaillaud M, 1967. Les Oligochètes de la savane de Gpakobo en Cote- d'Ivoire. Bulletin de l'Institut Francais d'Afrique Noire Douala 29 : 925-944.

Pagotto C, 1999. "Etude sur l'émission et le transfert dans les eaux et les sols des éléments traces métalliques et des hydrocarbures en domaine routier". Thèse de Doctorat. Université de Poitiers, $252 \mathrm{p}$.

Passinet JC, Dewailly E, Doucet-Leduc H, 2003. Contamination alimentaire. Environnement et santé publique-Fondements et pratiques 369$389 \mathrm{pp}$.

Pižl V. and Josens G, 1995. Earthworm communities along a gradient of urbanization. Environmental Pollution 90 : 7-14.

Promeyrat-Qotbi S, 2001. Contribution à l'étude de la pollution atmosphérique autoroutière : caractérisation des flux de déposition et de la contamination métallique de l'environnement de proximité, Thèse Université de Metz.

Public Eye investigation, 2016. DIRTY DIESEL - How Swiss Traders Flood Africa with Toxic Fuels, September 2016, $162 \mathrm{p}$.

Scott-Fordsmand JJ, Weeks JM, Hopkin SP, 1998. Toxicity of nickel to the earthworm and the applicability of the neutral red retention assay. Ecotoxicology 7: 291-295.

Scott-Fordsmand JJ, Weeks JM, Hopkin SP, 2000. Importance of contamination history for toxicity of copper. Environmental Toxicology and Chemistry 19: 1774-1780.

Spurgeon DJ. and Hopkin SP, 1995. Extrapolation of the laboratory-based OECD earthworm toxicity test to metal-contaminated field sites. Ecotoxicology 4: $190-205$.

Spurgeon DJ, Svendsen C, Rimmer VR, Hopkin SP, Weeks JM, 2000. Relative sensitivity of life-cycle and biomarker responses in four earthworm species exposed to zinc. Environmental Toxicology and Chemistry 19: 1800-1808. 
Ter braak CJK, 1986. Canonical correspondence analysis: a new eigenvector technique for multivariate direct gradient analysis. Ecology 67: 1167-1179.

Ter braak CJK, 1987. The analysis of vegetation environment relationships by canonical correspondence analysis. Vegetatio 69: 69-77.

Tong Z, Baldauf RW, Isakov V, Deshmukh P, Zhang $\mathrm{KM}, 2016$. Roadside vegetation barrier designs to mitigate near-road air pollution impacts. Science of the Total Environment 541: 920-927.

Trombulak SC. and Frissell CA, 2000. Review of ecological effects of roads on terrestrial and aquatic communities. Conservation Biology 14: 18-30.
Wcisło E, 1998. Soil Contamination with Polycyclic Aromatic Hydrocarbons (PAHs) in Poland - a Review. Polish Journal of Environmental Studies 7 (5); 267-272.

WHO (The World Health Organization), 2013. Access online: $\quad$ http://www.who.int/gho/phe/en/. Retrieved 6/01/2015.

Zheng Y, Wildonger JYB, Zhang Y, Kita A, Younger SH, Zimmerman S, Jan LY, Jan YN, 2008. Dynein is required for polarized dendritic transport and uniform microtubule orientation in axons. Nature Cell Biology 10: 1172-1180. 